Research Paper

\title{
Prognostic value of lymph nodes ratio in patients with stage III ovarian clear cell carcinoma: A retrospective study of patients in Southwest China
}

\author{
Dan $\mathrm{Ni}^{1,2}$, Xiguang $\mathrm{Mao}^{2}$, Zhengyu Li ${ }^{\circledR}$ \\ 1. Department of Obstetrics and Gynecology, Key Laboratory of Birth Defects and Related Diseases of Women and Children, Ministry of Education, West \\ China Second University Hospital, Sichuan University, Chengdu,610041, People's Republic of China. \\ 2. Department of Obstetrics and Gynecology, The affiliated hospital of Southwest Medical University, Luzhou,646000, People's Republic of China. \\ $\triangle$ Corresponding author: Zhengyu Li, M.D., West China Second University Hospital, Sichuan University, No. 20 Section 3, Renmin South Road, Chengdu, \\ Sichuan 610041, People's Republic of China. Tel: +86 18982151025; Fax: +86 (28) 85502391; Email: zhengyuli@scu.edu.cn \\ (1) The author(s). This is an open access article distributed under the terms of the Creative Commons Attribution License (https://creativecommons.org/licenses/by/4.0/). \\ See http://ivyspring.com/terms for full terms and conditions.
}

Received: 2018.09.12; Accepted: 2019.06.21; Published: 2019.08.19

\begin{abstract}
Background: Ovarian clear cell carcinoma (OCCC) has a worse prognosis compared to other histological subtypes. Although the survival effect of lymph nodes ratio (LNR) on ovarian carcinoma have been elucidated in several studies, the prognostic effect of LNR in OCCC has not been separately studied. This study aimed to investigate the prognostic significance of LNR in FIGO stage III OCCC.

Methods: Patients with FIGO stage III OCCC who underwent primary cytoreductive surgery and systematic lymphadenectomy from January 2008 to June 2014 in two independent hospitals were retrospectively reviewed. Two independent patients cohorts were used to investigate the survival impact of LNR by using Kaplan-Meier and Cox regression proportional hazard method.

Results: In training cohort, the 5 -year progression-free survival (PFS) rates was $32.4 \%$ for patients with LNR $\leq 25 \%$, and $19.8 \%$ for patients with LNR $>25 \%$, respectively $(p=0.017)$. The 5 -year overall survival (OS) rates was $41.3 \%$ for patients with LNR $\leq 25 \%$, and $25.8 \%$ for patients with LNR $>25 \%$, respectively $(p=0.003)$. In multivariate analysis, increased LNR was correlated with a poorer DFS (HR $=2.12,95 \% \mathrm{Cl}$ $1.32-3.41, \mathrm{p}=0.002)$ and $\mathrm{OS}(\mathrm{HR}=2.29,95 \% \mathrm{Cl} 1.37-5.12, \mathrm{p}=0.001)$. These results were verified in a validation cohort.
\end{abstract}

Conclusions: LNR is an independent survival predictor in patients with FIGO stage III OCCC.

Key words: ovarian clear cell carcinoma, lymph node ratio, survival

\section{Introduction}

Epithelial ovarian cancer (EOC) is a group of diseases with distinct clinical and histopathological features.[1, 2] Additionally, the incidence rate of lymph nodes (LNs) metastasis also differ in different EOC histological types and grades.[3-5] The standard management for advanced EOC is cytoreductive surgery and systematic lymphadenectomy followed by platinum-based and taxane-based chemotherapy $[6,7]$. Currently, the prognostic and therapeutic significance of systematic lymphadenectomy in EOC remains controversial[8]. However, LNs metastasis predicts poor survival in EOC patients has been well confirmed $[9,10]$.
Lymph node ratio (LNR), defined as the ratio of the number of metastatic lymph nodes (MLNs) to the number of resected lymph nodes (RLNs)[11], has been proved as an independent prognostic predictor in several malignancies including nonsmall cell lung cancer[12], breast cancer[13], cervical cancer[14], endometrial cancer[15], and EOC [8, 16-19]. However, the previous studies investigated the survival predictive value of LNR in all histologic subtypes of EOC and did not validate their results in another independent population.[8, 16-19]. In addition, the prognostic impact of LNR in ovarian clear cell carcinoma (OCCC), which accounts for 
approximately $5 \%$ to $25 \%$ of primary EOC, has not been separately clarified. [20] Furthermore, advanced OCCC, prone to chemo-resistant, has decreased survival compared with other histologic subtypes of EOC. [2, 20, 21] Moreover, there has no previous study assessed the prognostic role of LNR in Chinese population with advanced OCCC.

In the current study, we aimed to investigate the prognostic significance of LNR in two independent cohorts of Chinese patients with FIGO stage III OCCC.

\section{Methods}

\section{Patients selection}

OCCC patients who underwent surgical staging and lymphadenectomy in West China Second University Hospital, Sichuan University, and The Affiliated Hospital of Southwest Medical University from January 2008 to June 2014 were reviewed. Patients were enrolled into study according to the following inclusion criteria: (1) patients with a diagnosis of FIGO stage III; (2) patients underwent total hysterectomy, salpingo-oophorectomy, pelvic and para-aortic lymphadenectomy, omentectomy, and resected any suspicious and/or enlarged disease; (3) LNs metastasis positive; (4) no residual disease or residual disease $<1 \mathrm{~cm}$. Patients who had received neoadjuvant chemotherapy and interval debulking surgery were excluded from this study. The patient's age at diagnosis, clinicopathologic characters, treatment, and survival status was collected from the patients' medical records and clinical follow-up visits. 35 LNs was used as the cut-off value of systematic lymphadenectomy according to previous literature reported [21]. To investigate the survival impact of LNR, LNs positive patients were assigned into two groups according to reported [8]: LNR1 (LNR $\leq 25 \%$ ), and LNR2 (>25\%). The primary outcome was overall survival (OS) and progression-free survival (PFS). Patients from January 2008 to December 2012 was arranged in the training cohort, while patients from January 2013 to June 2014 was arranged in the validation cohort.

\section{Statistical analysis}

Correlations between categorical covariates were analyzed using chi-square test or Fisher's exact test. The PFS and OS curves were generated using Kaplan-Meier method and compared using log-rank test. Cox proportional hazard model was performed to assess the association between LNR and PFS and OS. SPSSTM, version 20.0 (SPSS Inc, Chicago, IL, USA) was used to performing the statistical analyses. $P$ values $<0.05$ were considered statistically significant.

\section{Results}

Patient baseline data in the training cohort are summarised in Table 1. The median patient age was 56 years old (30-89). The median follow up time was 40 months (1-119). The median number of RLNs was 46 (30-92). LN metastatic patients including 72 patients with both pelvic and para-aortic MLNs (40.4\%), 79 patients only have pelvic MLNs (44.4\%), and 27 patients only have para-aortic MLNs (15.2\%). The median number of total MLNs was 5 (1-69). The median number of pelvic MLNs and para-aortic MLNs was 4 (0-47) and $3(0-22)$, respectively. There were no significant differences between LNR and patient clinicopathologic characteristics in the training cohort (Table 2).

Table 1. Clinical characteristics of the patients in training and validation cohort

\begin{tabular}{|c|c|c|}
\hline \multirow[t]{2}{*}{ Patients characteristic } & Training cohort & Validation cohort \\
\hline & $\mathbf{N}=178(\%)$ & $\mathrm{N}=87(\%)$ \\
\hline Follow-up (month) & $40(1-119)$ & $38(2-72)$ \\
\hline Age (year) & $56(30-89)$ & $56(27-85)$ \\
\hline \multicolumn{3}{|l|}{ Grade } \\
\hline 2 & $47(26.4)$ & $9(10.3)$ \\
\hline 3 & $131(73.6)$ & $78(89.7)$ \\
\hline \multicolumn{3}{|l|}{ FIGO stage } \\
\hline IIIA1 & $39(21.9)$ & $3(3.4)$ \\
\hline IIIB & $15(8.4)$ & $7(8.1)$ \\
\hline IIIC & $124(69.7)$ & $77(88.5)$ \\
\hline \multicolumn{3}{|l|}{ Peritoneal cytology } \\
\hline Positive & $102(57.3)$ & $32(36.8)$ \\
\hline Negative & $54(30.3)$ & $38(43.6)$ \\
\hline Not available & $22(12.4)$ & $17(19.6)$ \\
\hline \multicolumn{3}{|l|}{ Residual disease after surgery } \\
\hline 0 & $82(46.1)$ & $36(41.4)$ \\
\hline $1-10 \mathrm{~mm}$ & $96(53.9)$ & $51(58.6)$ \\
\hline \multicolumn{3}{|c|}{ Number of resected lymph nodes } \\
\hline $\begin{array}{l}\text { Pelvic and para-aortic lymph } \\
\text { nodes }\end{array}$ & $46(30-92)$ & $34(23-106)$ \\
\hline Pelvic lymph nodes & $32(20-64)$ & $21(15-74)$ \\
\hline Para-aortic lymph nodes & $21(10-31)$ & $10(8-32)$ \\
\hline \multicolumn{3}{|c|}{ Patients with lymph nodes metastasis } \\
\hline $\begin{array}{l}\text { Only pelvic lymph nodes } \\
\text { metastasis }\end{array}$ & $79(44.4)$ & $52(59.8)$ \\
\hline $\begin{array}{l}\text { Only para-aortic lymph nodes } \\
\text { metastasis }\end{array}$ & $27(15.2)$ & $7(8)$ \\
\hline $\begin{array}{l}\text { Both pelvic and para-aortic } \\
\text { lymph nodes metastasis }\end{array}$ & $72(40.4)$ & $28(32.2)$ \\
\hline \multicolumn{3}{|c|}{ Number of metastatic lymph nodes } \\
\hline Pelvic lymph nodes & $4(0-47)$ & $2(0-35)$ \\
\hline Para-aortic lymph nodes & $3(0-22)$ & $3(0-29)$ \\
\hline $\begin{array}{l}\text { Pelvic lymph nodes and } \\
\text { para-aortic lymph nodes }\end{array}$ & $5(1-69)$ & $5(1-64)$ \\
\hline \multicolumn{3}{|l|}{ Lymph node ratio $(\%)$} \\
\hline$\leq 25$ & $106(59.6)$ & $49(56.3)$ \\
\hline$>25$ & $72(40.4)$ & $38(43.7)$ \\
\hline \multicolumn{3}{|l|}{ Adjuvant chemotherapy } \\
\hline Yes & $150(84.3)$ & $78(89.7)$ \\
\hline No & $28(15.7)$ & $9(10.3)$ \\
\hline \multicolumn{3}{|l|}{ Status } \\
\hline Alive & $60(33.7)$ & $41(47.1)$ \\
\hline Dead & $118(66.3)$ & $46(52.9)$ \\
\hline
\end{tabular}


Table 2. Correlation between LNR and clinicopathological characteristics in the training and validation cohort

\begin{tabular}{|c|c|c|c|c|c|c|c|c|c|c|}
\hline \multirow[t]{3}{*}{ Patient characteristics } & \multicolumn{5}{|c|}{ Training cohort } & \multicolumn{5}{|c|}{ Validation cohort } \\
\hline & \multicolumn{2}{|c|}{ LNR $\leq 25 \%$} & \multicolumn{2}{|c|}{ LNR > 25\% } & \multirow[t]{2}{*}{$P$} & \multicolumn{2}{|c|}{ LNR $\leq 25 \%$} & \multicolumn{2}{|c|}{ LNR $>25 \%$} & \multirow[t]{2}{*}{$P$} \\
\hline & $N=106$ & $\%$ & $\mathbf{N}=72$ & $\%$ & & $\mathbf{N}=49$ & $\%$ & $\mathbf{N}=$ & $\%$ & \\
\hline Median age, years (range) & \multicolumn{2}{|l|}{$57(30-83)$} & \multicolumn{2}{|c|}{$56(33-80)$} & 0.594 & \multicolumn{2}{|l|}{$54(29-85)$} & \multicolumn{2}{|c|}{$56(27-80)$} & 0.574 \\
\hline \multicolumn{11}{|l|}{ Grade } \\
\hline 2 & 31 & 29.2 & 16 & 22.2 & 0.387 & 6 & 12.2 & 3 & 7.9 & 0.509 \\
\hline 3 & 75 & 70.8 & 56 & 77.8 & & 43 & 87.8 & 35 & 92.1 & \\
\hline \multicolumn{11}{|l|}{ FIGO stage } \\
\hline IIIA1 & 20 & 18.9 & 19 & 26.4 & 0.029 & 2 & 4.1 & 1 & 2.6 & 0.742 \\
\hline IIIB & 5 & 4.7 & 10 & 13.9 & & 4 & 8.2 & 3 & 7.9 & \\
\hline IIIC & 81 & 76.4 & 43 & 59.7 & & 43 & 87.7 & 34 & 89.5 & \\
\hline \multicolumn{11}{|l|}{ Residual Disease } \\
\hline 0 & 48 & 45.3 & 34 & 47.2 & 0.799 & 19 & 38.8 & 17 & 44.7 & 0.576 \\
\hline $1-10 \mathrm{~mm}$ & 58 & 54.7 & 38 & 52.8 & & 30 & 61.2 & 21 & 55.3 & \\
\hline \multicolumn{11}{|l|}{ Peritoneal cytology* } \\
\hline Negative & 35 & 33 & 19 & 26.4 & 0.312 & 24 & 58.5 & 14 & 48.3 & 0.396 \\
\hline Positive & 49 & 46.2 & 53 & 73.6 & & 17 & 41.5 & 15 & 51.7 & \\
\hline \multicolumn{11}{|l|}{ Adjuvant chemotherapy } \\
\hline Yes & 89 & 84 & 61 & 84.7 & 0.891 & 45 & 91.8 & 33 & 86.8 & 0.448 \\
\hline No & 17 & 16 & 11 & 15.3 & & 4 & 8.2 & 5 & 13.2 & \\
\hline
\end{tabular}

"The patients with peritoneal cytology status not available were not included.

In the training patients cohort, the PFS and OS has no statistically significant difference in patients with $\geq 35$ RLNs and $<35$ RLNs $(\mathrm{p}=0.051, \mathrm{p}=0.07$; Figure 1). The median LNR was $8.7 \%(1.9 \%-72.7 \%)$. The median LNR was $6.7 \%(1.9 \%-7.6 \%)$ for stage IIIA1, $6.5 \%(2.1 \%-7.8 \%)$ for stage IIIB, and $9.1 \%$ $(1.9 \%-72.7 \%)$ for stage IIIC. The 5-year PFS rates in LNR1, LNR2 was $32.4 \%$, and $19.8 \%$ respectively $(\mathrm{p}=$ 0.017; Figure 2A).The 5-year OS rates in LNR1, LNR2 was $41.3 \%$, and $25.8 \%$ respectively $(p=0.003$; Figure 2B).

Further Cox univariate analysis revealed FIGO stage and LNR was related to PFS and OS. However, the residual tumor size was related to PFS, but not OS (Table 3). In multivariate analysis, the LNR was an independent predictor of PFS and OS (Table 3). OCCC patients in LNR2 group (LNR> 0.25) had an increased risk of relapse and mortality. The HR was 2.12 (95\% CI 1.32-3.41) for PFS and 2.29 (95\% CI 1.37-5.12) for OS (Table 3).

We further confirmed our results in the validation cohort. The patient baseline data and the association between the LNR and patient clinicopathologic characteristics also are showed in Table 1 and Table 2. The result indicated that the elevated LNR was correlated with worse PFS ( $p=$ $0.037)$ and $O S(p=0.011)$ (Figure 3). Univariate and multivariate analyses also proved the prognostic role of LNR (Table 4).

\section{Discussion}

In the current study, we verified that LNR is an independent survival predictor for FIGO stage III OCCC patients. Patients with elevated LNR (LNR> 0.25 ) have a worse PFS and OS.
Although systematic lymphadenectomy is essential to establish stage of EOC, its therapeutic role in advanced ovarian cancer still controversial.[22-25] Recently, randomized controlled studies revealed that patients with EOC did not gain a survival benefit from systematic lymphadenectomy.[22, 24, 26] However, systematic lymphadenectomy might improve OCCC patients survival through remove of chemo-resistant metastatic LNs.[2] Therefore, advanced OCCC patients might benefit from systematic lymphadenectomy.

A

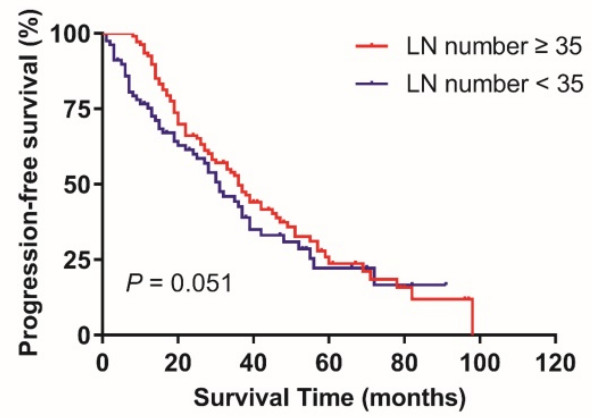

B

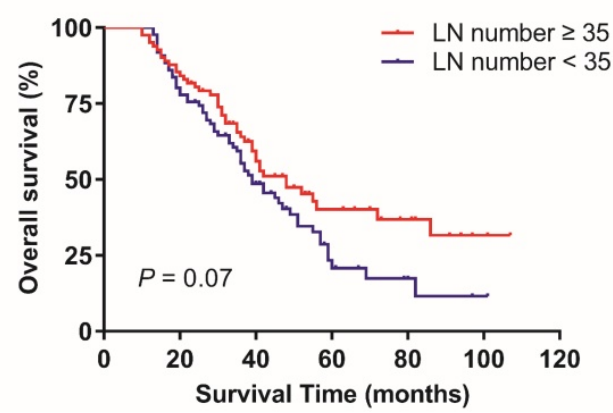

Figure 1. Effect of the number of resected lymph nodes on progression-free survival (A) and overall survival (B). Kaplan-Meier. 


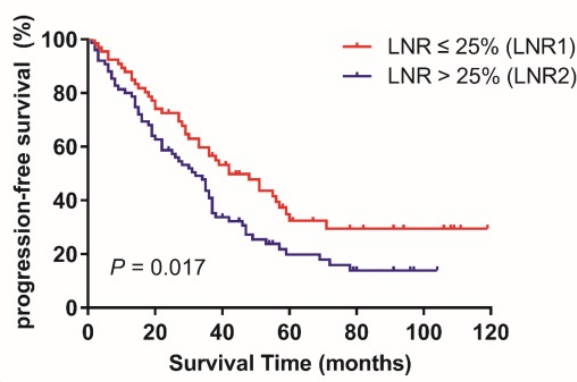

B

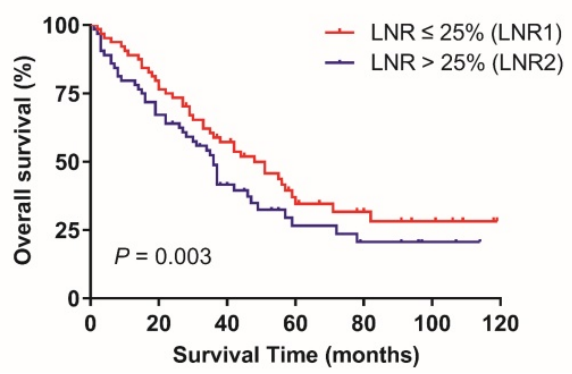

Figure 2. Effect of LNR (lymph node ratio) on progression-free survival (A) and overall survival $(B)$ in training cohort. Kaplan-Meier.
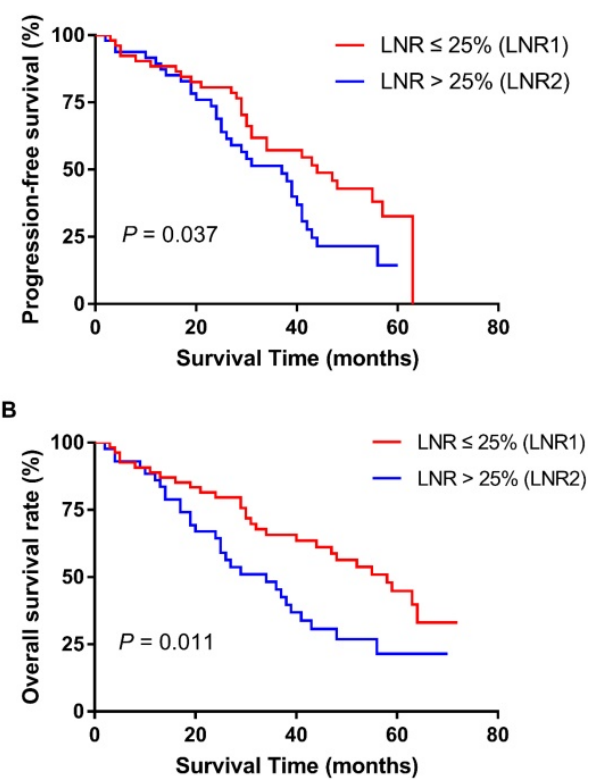

Figure 3. Effect of LNR (lymph node ratio) on progression-free survival (A) and overall survival (B) in validation cohort. Kaplan-Meier.

Table 3. Univariate and multivariate analyses of patients for OS and DFS in training cohort

\begin{tabular}{|c|c|c|c|c|c|c|c|c|c|c|c|c|}
\hline \multirow[t]{3}{*}{ Characteristic } & \multicolumn{6}{|l|}{ OS } & \multicolumn{6}{|l|}{ DFS } \\
\hline & \multicolumn{3}{|c|}{ Univariate analyses } & \multicolumn{3}{|c|}{ Multivariate analyses } & \multicolumn{3}{|c|}{ Univariate analyses } & \multicolumn{3}{|c|}{ Multivariate analyses } \\
\hline & HR & $95 \% \mathrm{CI}$ & $P$ & HR & $95 \% \mathrm{CI}$ & $P$ & HR & $95 \% \mathrm{CI}$ & $P$ & HR & $95 \% \mathrm{CI}$ & $P$ \\
\hline \multicolumn{13}{|l|}{ Grade } \\
\hline 2 & 1 & & & & & & 1 & & & & & \\
\hline 3 & 1.24 & $0.78-2.13$ & 0.314 & & & & 1.39 & $0.87-2.08$ & 0.746 & & & \\
\hline \multicolumn{13}{|c|}{ Peritoneal cytology } \\
\hline Negative & 1 & & & & & & 1 & & & & & \\
\hline Positive & 1.19 & $0.63-2.22$ & 0.592 & & & & 1.37 & $0.81-2.32$ & 0.235 & & & \\
\hline \multicolumn{13}{|c|}{ Residual disease } \\
\hline 0 & 1 & & & & & & 1 & & & 1 & & \\
\hline $1-10 \mathrm{~mm}$ & 1.43 & $0.63-3.25$ & 0.393 & & & & 1.31 & $1.03-2.76$ & 0.001 & 1.3 & $1.04-2.43$ & 0.023 \\
\hline \multicolumn{13}{|l|}{ FIGO stage } \\
\hline IIIA1 vs. IIIB & 1.73 & $1.12-2.62$ & 0.003 & 1.71 & $0.45-3.22$ & 0.242 & 1.42 & $1.01-1.73$ & 0.016 & 1.23 & $0.63-1.68$ & 0.55 \\
\hline IIIA1 vs. IIIC & 2.91 & $1.94-8.94$ & 0.014 & 2.83 & $0.78-7.42$ & 0.381 & 1.66 & $1.16-2.18$ & 0.025 & 1.46 & $0.77-1.96$ & 0.319 \\
\hline \multicolumn{13}{|c|}{ Lymph node ratio (LNR) } \\
\hline$\leq 25 \%$ & 1 & & & 1 & & & 1 & & & 1 & & \\
\hline$>25 \%$ & 2.92 & $1.45-4.89$ & $<0.001$ & 2.29 & $1.37-5.12$ & 0.001 & 2.52 & $1.56-4.07$ & $<0.001$ & 2.12 & $1.32-3.41$ & 0.002 \\
\hline
\end{tabular}

Table 4. Univariate and multivariate analyses of patients for OS and DFS in validation cohort

\begin{tabular}{|c|c|c|c|c|c|c|c|c|c|c|c|c|}
\hline \multirow[t]{3}{*}{ Characteristic } & \multicolumn{6}{|l|}{ OS } & \multicolumn{6}{|l|}{ DFS } \\
\hline & \multicolumn{3}{|c|}{ Univariate analyses } & \multicolumn{3}{|c|}{ Multivariate analyses } & \multicolumn{3}{|c|}{ Univariate analyses } & \multicolumn{3}{|c|}{ Multivariate analyses } \\
\hline & HR & $95 \% \mathrm{CI}$ & $P$ & HR & $95 \% \mathrm{CI}$ & $P$ & HR & $95 \% \mathrm{CI}$ & $P$ & HR & $95 \% \mathrm{CI}$ & $P$ \\
\hline \multicolumn{13}{|l|}{ Grade } \\
\hline 2 & 1 & & & & & & 1 & & & & & \\
\hline 3 & 1.03 & $0.68-1.4$ & 0.876 & & & & 1.07 & $0.81-1.73$ & 0.373 & & & \\
\hline \multicolumn{13}{|c|}{ Peritoneal cytology } \\
\hline Negative & 1 & & & & & & 1 & & & & & \\
\hline Positive & 1.31 & $0.91-1.88$ & 0.149 & & & & 1.15 & $0.79-1.68$ & 0.471 & & & \\
\hline \multicolumn{13}{|c|}{ Residual disease } \\
\hline 0 & 1 & & & 1 & & & 1 & & & 1 & & \\
\hline $1-10 \mathrm{~mm}$ & 1.44 & $1.13-1.83$ & 0.013 & 1.67 & $1.19-2.34$ & 0.038 & 1.81 & $1.48-2.20$ & 0.001 & 1.90 & $1.39-3.74$ & 0.03 \\
\hline \multicolumn{13}{|l|}{ FIGO stage } \\
\hline IIIA1 vs. IIIB & 1.2 & $0.65-2.23$ & 0.599 & & & & 1.066 & $0.66-1.71$ & 0.814 & & & \\
\hline IIIA1 vs. IIIC & 1.39 & $0.78-1.42$ & 0.265 & & & & 1.13 & $0.71-1.8$ & 0.602 & & & \\
\hline \multicolumn{13}{|c|}{ Lymph node ratio (LNR) } \\
\hline$\leq 25 \%$ & 1 & & & 1 & & & 1 & & & 1 & & \\
\hline$>25 \%$ & 3.35 & $1.87-5.98$ & $<0.001$ & 2.80 & $1.97-3.96$ & 0.001 & 1.99 & $1.31-3.01$ & $<0.001$ & 1.96 & $1.44-2.68$ & $<0.001$ \\
\hline
\end{tabular}


The optimal lymphadenectomy cut-off value in OCCC patients has not been defined. Takei et al. showed that patients with $\geq 35$ LNs removed have an improved recurrence-free survival.[21] Pereira et al. study defined optimal lymphadenectomy cut-off value was at least obtain 15 pelvic LNs and 7 aortic LNs.[27] However, the number of metastatic LNs is depended on many factors such as the surgeon and the pathologist's distinct experience in searching for positive LNs, the patients' anatomic variation, the extent of the tumor, and patients'age.[16, 17] Hence, the limitation of use LNs status to predict survival might be addressed by using LNR.

The prognostic role of LNR has been discussed in advanced EOC. Ataseven et al. [8] found the 5-year OS rates were higher in patients with LNR $\leq 0.25$ compared to patients with LNR > $0.25 \quad(42.5 \%$ vs.18.0\%). Ayhan et al. [17] focused on the FIGO stage III high-grade ovarian serous carcinoma (HGOSC), which also have a poor prognosis. They found the 5 -year OS was decreased from $65.1 \%$ in LNR1 $(<10 \%)$ to $42.5 \%$ in LNR2 $(10 \% \leq \mathrm{LNR}<50 \%)$, and to $25.6 \%$ in LNR3( $\geq 50 \%)$, LNR also was an independent survival predictor for OS. In the current study, we proved LNR was an independent predictor for decreased PFS and OS in FIGO stage III OCCC patients. These results validated the feasibility of use LNR to predict prognosis in FIGO stage III OCCC.

The LNR cut-off point used to assign patients to a lower or higher LNRs group has not been well defined. [8, 16-18] In this study, we used the LNR cut-off value described by Ataseven et al. [8]. Further studies are needed to establish a standard LNR cut-off point. In addition, in the lymphadenectomy in ovarian neoplasms (LION) study, although 56\% EOC patients had LNs micro-metastases, systematic lymphadenectomy offers no benefit to patients who underwent maximum or optimal cytoreduction and had clinically and radiologic negative lymph nodes.[28] In our study, we defined optimal lymphadenectomy as the LION study described.[28] The result indicated that LNR might an independent predictor for worse OS and PFS in FIGO stage III OCCC patients with LNs metastasis. However, the resected LN number did not have survival effects in patients with OCCC.

Compared to previous studies, our study has several advantages. Above all, this is the first study investigating the prognostic role of LNR in OCCC based on Chinese population. Previous studies all based on European population [8, 17] or the SEER (Surveillance, Epidemiology, and End Results) database from the United States. [16, 18, 19] The postoperative adjuvant therapy information does not provide in SEER. Secondly, this study mainly focused on the prognostic value of LNR in OCCC, since OCCC has a poorer prognosis than other histological subtypes. Thirdly, previous studies did not validate their findings and conclusions using an independent validation cohort.[11-15] In order to strengthen the credibility of our study, two independent patients cohorts were used to assess the prognostic role of LNR, and both patients cohort proved the prognostic value of LNR. However, our findings should be validated in future prospective study.

\section{Conclusions}

LNR has a significant impact on PFS and OS and might be used as a predictor of survival in patients with advanced OCCC. However, these findings need to be verified in future prospective studies.

\section{Acknowledgements}

Z.L. and D.N. conceived and designed this study. D.N. and X.M. collected and analyzed the data. D.N. wrote the manuscript. All authors approved the final manuscript.

\section{Competing Interests}

The authors have declared that no competing interest exists.

\section{References}

1. Köbel M, Kalloger SE, Boyd N, et al. Ovarian carcinoma subtypes are different diseases: implications for biomarker studies. PLoS Med. 2008. 5(12): e232.

2. Ye S, Yang J, You Y, et al. Comparison of Clinical Characteristic and Prognosis between Ovarian Clear Cell Carcinoma and Serous Carcinoma: A 10-Year Cohort Study of Chinese Patients. PLoS One. 2015;10:e133498.

3. Powless CA, Aletti GD, Bakkum-Gamez JN, Cliby WA. Risk factors for lymph node metastasis in apparent early-stage epithelial ovarian cancer: implications for surgical staging. Gynecol Oncol. 2011;122:536-540.

4. Kleppe M, van der Aa MA, Van Gorp T, Slangen BF, Kruitwagen RF. The impact of lymph node dissection and adjuvant chemotherapy on survival: A nationwide cohort study of patients with clinical early-stage ovarian cancer. Eur J Cancer. 2016;66:83-90.

5. Takeshima N, Hirai Y, Umayahara K, Fujiwara K, Takizawa K, Hasumi K. Lymph node metastasis in ovarian cancer: difference between serous and non-serous primary tumors. Gynecol Oncol. 2005;99:427-431.

6. Narasimhulu DM, Khoury-Collado F, Chi DS. Radical surgery in ovarian cancer. Curr Oncol Rep. 2015;17:16.

7. Chang SJ, Bristow RE, Ryu HS. Prognostic significance of systematic lymphadenectomy as part of primary debulking surgery in patients with advanced ovarian cancer. Gynecol Oncol. 2012; 126: 381-6.

8. Ataseven B, Grimm C, Harter P, et al. Prognostic value of lymph node ratio in patients with advanced epithelial ovarian cancer. Gynecol Oncol. 2014. 135(3): 435-40.

9. Trimbos JB. Lymphadenectomy in ovarian cancer: standard of care or unnecessary risk. Curr Opin Oncol. 2011. 23(5): 507-11.

10. Mahdi $\mathrm{H}$, Moslemi-Kebria $\mathrm{M}$, Levinson $\mathrm{KL}$, et al. Prevalence and prognostic impact of lymphadenectomy and lymph node metastasis in clinically early-stage ovarian clear cell carcinoma. Int J Gynecol Cancer. 2013. 23(7): 1226-30.

11. Lee SG, Ho J, Choi JB, Kim TH, Kim MJ, Ban EJ, et al. Optimal Cut-Off Values of Lymph Node Ratio Predicting Recurrence in Papillary Thyroid Cancer. Medicine (Baltimore). 2016; 95: e2692.

12. Jonnalagadda S, Arcinega J, Smith C, Wisnivesky JP. Validation of the lymph node ratio as a prognostic factor in patients with N1 nonsmall cell lung cancer. Cancer. 2011; 117: 4724-31.

13. van der Wal BC, Butzelaar RM, van der Meij S, Boermeester MA. Axillary lymph node ratio and total number of removed lymph nodes: predictors of survival in stage I and II breast cancer. Eur J Surg Oncol. 2002; 28: 481-9.

14. Chen Y, Zhang L, Tian J, Fu X, Ren X, Hao Q. Significance of the absolute number and ratio of metastatic lymph nodes in predicting postoperative 
survival for the International Federation of Gynecology and Obstetrics stage IA2 to IIA cervical cancer. Int J Gynecol Cancer. 2013. 23(1): 157-63.

15. Polterauer S, Khalil S, Zivanovic O, et al. Prognostic value of lymph node ratio and clinicopathologic parameters in patients diagnosed with stage IIIC endometrial cancer. Obstet Gynecol. 2012. 119(6): 1210-8.

16. Mahdi H, Thrall M, Kumar S, et al. The prognostic impact of the ratio of positive lymph nodes on survival of epithelial ovarian cancer patients. J Surg Oncol. 2011. 103(7): 724-9.

17. Ayhan A, Ozkan NT, Sarı ME, et al. Impact of lymph node ratio on survival in stage III ovarian high-grade serous cancer: a Turkish Gynecologic Oncology Group study. J Gynecol Oncol. 2018. 29(1): e12.

18. Zhou J, He ZY, Li FY, et al. Prognostic value of lymph node ratio in stage IIIC epithelial ovarian cancer with node-positive in a SEER population-based study. Oncotarget. 2016. 7(7): 7952-9.

19. Wang J, Li J, Chen R, Lu X. Survival effect of different lymph node staging methods on ovarian cancer: An analysis of 10878 patients. Cancer Med. 2018; 7: 4315-29.

20. del CMG, Birrer M, Schorge JO. Clear cell carcinoma of the ovary: a review of the literature. Gynecol Oncol. 2012. 126(3): 481-90.

21. Takei $Y$, Takahashi S, Machida S, et al. Impact of the number of removed lymph nodes on recurrence-free survival in stage I ovarian clear cell carcinoma. Int J Clin Oncol. 2018

22. Panici PB, Maggioni A, Hacker N, et al. Systematic aortic and pelvic lymphadenectomy versus resection of bulky nodes only in optimally debulked advanced ovarian cancer: a randomized clinical trial. J Natl Cancer Inst. 2005. 97(8): 560-6.

23. Chan JK, Urban R, Hu JM, et al. The potential therapeutic role of lymph node resection in epithelial ovarian cancer: a study of 13918 patients. Br J Cancer. 2007. 96(12): 1817-22

24. Maggioni A, Benedetti PP, Dell'Anna T, et al. Randomised study of systematic lymphadenectomy in patients with epithelial ovarian cancer macroscopically confined to the pelvis. Br J Cancer. 2006. 95(6): 699-704.

25. du BA, Reuss A, Harter P, Pujade-Lauraine E, Ray-Coquard I, Pfisterer J. Potential role of lymphadenectomy in advanced ovarian cancer: a combined exploratory analysis of three prospectively randomized phase III multicenter trials. J Clin Oncol. 2010. 28(10): 1733-9.

26. Gockley A, Melamed A, Bregar AJ, et al. Outcomes of Women With High-Grade and Low-Grade Advanced-Stage Serous Epithelial Ovarian Cancer. Obstet Gynecol. 2017. 129(3): 439-447.

27. Pereira A, Irishina N, Pérez-Medina $\mathrm{T}$, et al. Defining the optimal lymphadenectomy cut-off value in epithelial ovarian cancer staging surgery utilizing a mathematical model of validation. Eur J Surg Oncol. 2013;39:290-296.

28. Harter P, Sehouli J, Lorusso D et al. LION:Lymphadenectomy in ovarian neoplasms - a prospective randomized AGO study group led gynecologic cancer intergroup trial. J Clin Oncol. 2017; 35: 5500-5500. 